\title{
Patente Provisional en Chile: Procedimiento, Ventajas e Incentivos Para Innovar
}

\author{
Leonardo Javier Castillo-Cardenas ${ }^{1^{*}}$, Carlos Andrés Domínguez-Scheid ${ }^{2}$
}

\begin{abstract}
Provisional patent in Chile: Procedure, advantages, and incentives to innovate
The production of knowledge and information results in the improvement of living standards. Economic development has determined that the producers of this knowledge must be adequately rewarded for their inventions, considering that innovation and the solution for problems that affect people's productivity and quality of life must be protected. These rewards include property rights granted by an invention patent. However, in the invention process, there is a time when the inventor needs testing or experimenting for his innovation, and it will become public without the protection of a final patent application. In this situation and to protect the creative process, it is functional to introduce a type of provisional patent. It will last for a while until the request for a nonprovisional patent. This article analyzes the provisional patent and its re-introduction into the Chilean law of intellectual property from a comparative and historical perspective.
\end{abstract}

Keywords: Provisional patent; nonprovisional patent; Intellectual Property Rights; Chile, Comparative Economic Law

Resumen: La producción de conocimiento e información deviene en la mejora de los estándares de vida. El desarrollo económico ha determinado que los productores de este conocimiento deben ser recompensados adecuadamente por sus invenciones, ya que de esa manera se promueve la innovación y la solución de problemas que afectan a la productividad y a la calidad de vida de las personas. Dentro de estas recompensas aparecen los derechos de propiedad que se otorgan mediante una patente de invención. Sin embargo, durante el desarrollo del proceso de invención hay un momento en que el inventor está en la necesidad de probar o experimentar su innovación, para lo que se enfrenta a la necesidad de hacerla pública, sin que su desarrollo le permita presentar una solicitud de patente definitiva. Para esta situación, y con la finalidad de proteger su proceso creativo, se consideran útil un tipo de patente provisional, que durará un tiempo hasta que pueda pedir su patente definitiva. Este artículo analiza la patente provisional y su re-introducción en el sistema chileno de propiedad intelectual, desde una perspectiva comparada e histórica.

Palabras clave: Patentes provisionales; patentes precaucionales; certificados de protección; propiedad intelectual Chile; Derecho Económico Comparado

Submitted: September $1^{\text {st }}, 2019$ / Approved: August $30^{\text {th }}, 2021$

\section{Introducción}

En Chile el organismo encargado de recibir las solicitudes de patente y de tramitarlas es el Instituto Nacional de Propiedad Industrial (INAPI) creado por la ley 20.254 de 2008. Dentro de esta protección a la propiedad intelectual las patentes cumplen un rol fundamental. Entre los distintos tipos de patente existe la patente provisional, que es aquella que permite resguardar la novedad de la invención y amparar transitoriamente los derechos de los inventores solicitantes contra posibles usurpaciones. En Chile fue reconocida hasta el año 2005, y recientemente, ha sido reestablecida en la ley. Esta nueva patente provisional tiene su origen en un proyecto de ley presentado el año 2018, hoy ley 21.335, promulgada el 22 de junio de 2021 y publicada el 05 de julio de 2021, el que entre otros aspectos relativos a la propiedad industrial, propone el re-establecimiento de las patentes provisionales o precaucionales.

Considerando la importancia de la propiedad industrial, esencial para compatibilizar la certeza que requiere el entorno de negocios con la necesidad de proteger las innovaciones, se parte de la premisa de que el establecimiento de las patentes provisionales protege los intereses del inventor que no desea que terceros (polizones o free riders), sin invertir ni arriesgar, quieran aprovecharse de sus beneficios ${ }^{1}$. En el presente artículo mediante tanto un análisis histórico de la regulación en Chile, como un análisis dogmático de la figura, así como su consagración en diferentes legislaciones de diversas latitudes, se estudia el procedimiento para la obtención de la patente provisional para concluir las ventajas que de ella devienen.

\section{Contexto}

En la época moderna, el desarrollo de la protección a la propiedad intelectual se remonta a la Constitución de Estados Unidos, vigente desde 1787. El artículo 1.8 de la ley fundamental americana dispone que el Congreso de ese país está facultado "Para fomentar el progreso de la ciencia y las artes útiles, asegurando a los autores e inventores, por un tiempo limitado, el derecho exclusivo sobre sus respectivos escritos y descubrimientos" (Samuelson y Nordhaus, p. 228). En Chile, la protección de este tipo de propiedad ha sido reconocida en las constituciones de 1833, 1925 y 1980 (Larraguibel, 1979, p. 7). Como plantea Ruiz-Tagle (2004, p. 63), tradicionalmente se distinguen dos tesis para justificar el privilegio de patentes. La primera de estas,

(1) Juridical Sciences Department, Universidad de La Frontera

(2) Universidad Católica de Temuco, Departamento de Ciencias Jurídicas

*Autor de correspondencia: leonardo.castillo@ufrontera.cl

${ }^{1}$ Si bien quienes están en contra pudieran sostener que aquello importa incentivos negativos que decantaran en atentados contra la libre competencia, dicha cuestión debiera resolverse conforme a las normas específicas de dicha materia, lo que escapa de las finalidades de este artículo. 
denominada "tesis de incentivo-lucro-monopolio" plantea que el medio más eficiente para el logro de las invenciones necesarias para el desarrollo económico es el otorgamiento de derechos monopólicos o exclusivos. La segunda denominada "intercambio de secretos" plantea que la patente sería el medio idóneo para la divulgación tecnológica, toda vez que sin ella las invenciones permanecerían lejos del conocimiento público. Señalemos al respecto que la abolición de las patentes llevaría a los innovadores a optar sistemáticamente por el secreto de fabricación con el riesgo de integrarse verticalmente en unidades de produccion si no son ellos los fabricantes (Tirole, 2017, p. 462). D'Amore (2015, pp 7-9) complementa distinguiendo entre distintas teorías para justificar los derechos de propiedad intelectual: aquellas que apelan al derecho natural, las pragmáticas y las utilitaristas, donde la primera pone énfasis en la relación del autor con su obra; la segunda en que los inventores han de ser compensados por los riesgos y por la inversión de tiempo y esfuerzo que hayan realizado; y finalmente aquella que ve a los derechos como títulos e instrumentos de mercado en una, y como un medio para la obtención de otra patente de propiedad de un tercero mediante el intercambio en otra. La solución del conflicto entre los incentivos a la inversión y el poder de mercado está dada por una solución ecléctica que no es otra que otorgarles duración limitada a las patentes (Coloma, p. 189)

El Código Civil chileno, vigente desde 1855, consagra en su artículo 584 que "Las producciones del talento o del ingenio son una propiedad de sus autores", y que ese tipo de propiedad será regido por leyes especiales. Samuelson y Nordhaus (2010) sostienen que "la legislación especial que regula las patentes, [...] crea derechos de propiedad intelectual. El propósito es conceder al propietario una protección especial contra la copia y uso del material por otros, sin compensar al propietario o creador original". Respecto de la duración de las patentes, en palabras de Cooter y Ulen (2016, p. 172), al establecer un monopolio de carácter temporal destinado a recompensar al inventor y gravar al comprador, "la vida óptima de una patente alcanza un mejor equilibrio entre el estímulo de la creatividad y el desaliento de la difusión”.

Coincidimos con Botana Agra (2017, p. 99) en que "el sistema de patentes constituye un instrumento básico para el impulso del desarrolllo económico y un elemento fundamental para el fomento de la investigación e innovación en el ámbito de la técnica”. En términos similares Bercovitz (2017, p. 477) señala que "el derecho de patentes sirve para promover el progreso tecnológico e industrial dentro de un marco de libre competencia, y esa libre competencia no es posible en el ámbito tecnológico si no existiera el derecho de patentes". Clarke (1993, p. 208) complementa esta idea apuntando dos razones que hacen de la investigación un proceso económico especial. La primera, toda vez que por una parte tiene un alto grado de incertidumbre,y por otro por que su producto, esto es, la información, tiene características propias de un bien público.

El concepto de propiedad intelectual comprende tanto los derechos de autor como la propiedad industrial, "que incluye las patentes de invención, los modelos de utilidad, los dibujos y diseños industriales, las marcas, indicaciones geográficas y de origen, circuitos integrados, entre otros" (Burgos, 2014, p. 43). Tirole (2017, p.461) sitúa las primeras patentes en la Grecia de la antigüedad y luego, durante el siglo XV, en las repúblicas italianas de Florencia y Venecia. Esas tres sociedades tienen una característica común: Tuvieron un rol hegemónico en el mundo antiguo y el de fines de la Edad Media, respectivamente. De esta manera, las patentes contribuyen al desarrollo económico y son poderosos incentivos para quienes invierten en crear conocimiento. Para que una invención sea patentable debe cumplir con ciertos requisitos: Debe ser nueva, implicar actividad inventiva y ser susceptible de aplicación industrial. De Souza Querido et al. (2011) explican que el poder de una patente se expresa cuando el producto o proceso innovador sale al mercado y el derecho exclusivo garantizado por la patente genera poder de mercado y la posibilidad para el titular de obtener una ventaja competitiva sobre sus competidores. Antes de salir al mercado, la investigación y desarrollo puede requerir de la exposición al público. Este es el contexto en que aparece la utilidad de las llamadas patentes precaucionales o previsionales. Como señala Cortés (2012, p. 249) es en ese período donde "la explotación de la invención presenta su máxima rentabilidad, pues el ritmo de desarrollo técnico contemporáneo implica que sean las nuevas invenciones o desarrollos tecnológicos los que destruyen el valor económico de las invenciones preexistentes". Este tipo de patente no es extraña para el ordenamiento chileno. Al respecto, Claro Solar (1992) explicaba que:

"Dentro del examen previo del invento o descubrimiento que consigna la ley chilena para establecer la novedad, el otorgamiento de la patente precaucional tiene la ventaja de proteger los derechos del descubridor o inventor sin obligarlo a entrar a hacer los gastos que exige la tramitación de su solicitud que no podría paralizar sin exponer a la caducidad de su derecho. Por lo demás, la patente precaucional solamente ampara el derecho preferente del dueño de ella para que se le otorgue a él la patente de invención, si durante el año se presentara otra persona solicitando patente para una invención igual o para idéntico descubrimiento". (p. 612)

\section{Evolución de la regulacion de las patentes provisionales o pre- caucionales en Chile.}

El Decreto 588 de 29 de septiembre de 1925, que como señala GarcíaHuidóbro (1992, p. 33) fue el primer texto de la Ley sobre Propiedad Industrial, disponía en su artículo 13 que cualquier inventor domiciliado en el pais que tuviese una invencion en estudio y que necesitase practicar esperiencias o hacer construir algun mecanismo o aparato el cual le obligue a hacer pública su idea, podrá amparar de manera transitoria sus derechos contra posibles usurpaciones, pidiendo un certificado de protección o "patente precaucional" al órgano público competente -en este caso la Oficina de Propiedad Industrial, que dependía del Ministerio de Agricultura e Industria- la que se la otorgará por el término de un año, previo pago del impuesto que para este documento se establecía. Este certificado le daba al inventor -que aquí denomina poseedor- un derecho legal preferente sobre cualquiera otra persona que durante el año de protección pretendiese solicitar privilegio sobre la misma materia. Si el poseedor de una patente precaucional dejaba transcurrir ese año sin haber solicitado la patente definitiva, la ley disponía que el invento pasaría a ser de uso común. 
Finalmente, en casos especiales justificados, calificados por la Oficina se establecía que la patente precaucional podría renovarse por un segundo año, debiendo el interesado abonar un impuesto equivalente a tres veces la suma pagada por el primer año.

El Decreto 588 fue reemplazado en el año 1931 por el Decreto Ley $\mathrm{N}^{\circ}$ 958, de 27 de julio, "Ley sobre propiedad industrial". Analizando el texto publicado en el Repertorio de Legislación y Jurisprudencia (1993, p. 16) se aprecia que la nueva Ley mantuvo la patente precaucional en el mismo artículo 13, modificando la competencia de la Oficina por el flamante Departamento de Industrias fabriles, dependiente del Ministerio de Fomento. También pasa a denominar al inventor como dueño de la patente precaucional, a diferencia de la ley anterior que lo llamaba poseedor.

Esta ley fue reemplazada por la ley $\mathrm{N}^{\circ} 19.039$, sobre privilegios industriales y protección de los derechos de propiedad industrial, publicada el 25 de enero de 1991. Analizaremos su texto original, de acuerdo al Repertorio de Legislación y Jurisprudencia (1993, p. 33), el que en su artículo 42 introducía las siguientes innovaciones: En primer término, el pago de impuesto es reemplazado por pago del derecho. En segundo lugar, el concepto de "uso común" es sustituido por el de "dominio público". En tercer lugar se elimina la posibilidad de pedir una prórroga por un segundo año, y, finalmente en cuarto lugar, se establece que el plazo de duración de la patente definitiva se contará desde la fecha en que se haya otorgado la patente precaucional.
El 04 octubre de 1999 fue presentado un proyecto de ley², mediante mensaje del Presidente de la República, que buscaba modificar la ley de propiedad industrial. Entre otras modificaciones y argumentando como motivo la adecuación de legislación nacional con los Aspectos de los Derechos de Propiedad Intelectual relacionados con el Comercio (en español ADPIC, en inglés TRIPS) en materia de patentes de invención y procedimientos de concesión, se proponía la eliminación de las patentes precaucionales. En la historia de la ley no figura ninguna justificación más allá de aquella, adoleciendo de debate la tramitación.

El proyecto resultó aprobado el 11 de marzo de 2005, transformándose en la Ley $\mathrm{N}^{\circ}$ 19.996, que modificó el texto de la ley 19.039 de propiedad industrial eliminando el contenido del artículo 42 que contemplaba la patente precaucional, y reemplazándolo por otro que contempla las divulgaciones inocuas ${ }^{3}$. Este tipo de divulgación se relaciona con el requisito de novedad que se exige para la patentabilidad de una invención, en el sentido de que no constituirá un impedimento para obtener una patente el que la invención haya sido conocida públicamente antes de que se solicite su registro. Esta excepción opera solamente en los casos señalados en la ley, que considera a las prácticas, ensayos y construcción de mecanismos o aparatos que deba hacer el solicitante que tenga una invención en estudio, o a las exhibiciones del invento hechas por el solicitante en exposiciones oficiales u oficialmente reconocidas y los abusos y las prácticas desleales de las que hubiese sido objeto el solicitante.

Cuadro de la patente precaucional en las distintas leyes chilenas

\begin{tabular}{|c|c|c|c|c|c|}
\hline Tipo de norma & Nombre & Artículo & Denominación & Plazo de la protección & Prórroga \\
\hline $\begin{array}{l}\text { Decreto Ley } 588 \text { de } 29 \text { de } \\
\text { septiembre de } 1925\end{array}$ & $\begin{array}{l}\text { Sobre organización de los servicios } \\
\text { de la propiedad industrial }\end{array}$ & Artículo 13 & $\begin{array}{l}\text { Certificado de protec- } \\
\text { cion o "patente precau- } \\
\text { cional" }\end{array}$ & Un año & Si, hasta por un año más \\
\hline $\begin{array}{l}\text { Decreto con Fuerza de Ley } \\
958 \text { de } 27 \text { de julio de } 1931\end{array}$ & Ley sobre propiedad industrial & Artículo 13 & $\begin{array}{l}\text { Certificado de protec- } \\
\text { cion o patente precau- } \\
\text { cional }\end{array}$ & Un año & $\mathrm{Si}$, hasta por un año más \\
\hline $\begin{array}{l}\text { Ley } 19.039 \text { de } 25 \text { de enero } \\
\text { de } 1991\end{array}$ & $\begin{array}{l}\text { Establece normas aplicables a los } \\
\text { privilegios industriales y protec- } \\
\text { ción de los derechos de propiedad } \\
\text { industrial }\end{array}$ & Artículo 42 & $\begin{array}{l}\text { Certificado de protec- } \\
\text { ción o patente precau- } \\
\text { cional }\end{array}$ & Un año & No \\
\hline $\begin{array}{l}\text { Decreto con Fuerza de } \\
\text { Ley No } 3 \text { del } 20 \text { de junio } \\
\text { de } 2006 \\
\text { (Ley vigente) }\end{array}$ & $\begin{array}{l}\text { Fija texto refundido, coordinado y } \\
\text { sistematizado de la ley de propie- } \\
\text { dad industrial }\end{array}$ & No & No & No & No \\
\hline $\begin{array}{l}\text { Ley } \mathrm{N}^{\circ} 21.335 \text {, del } 5 \text { de ju- } \\
\text { lio de } 2021 \\
\text { (Entrará en vigencia una } \\
\text { vez que se publique en el } \\
\text { Diario Oficial su regla- } \\
\text { mento) }\end{array}$ & $\begin{array}{l}\text { Modifica la ley } \mathrm{N}^{\circ} 19.039 \text {, de pro- } \\
\text { piedad industrial y la ley } \mathrm{N}^{\circ} 20.254 \text {, } \\
\text { que establece el Instituto Nacional } \\
\text { de Propiedad Industrial }\end{array}$ & Art. 40 & Patente provisional & $\begin{array}{l}\text { Doce meses, contado des- } \\
\text { de la fecha de presentación } \\
\text { de la solicitud provisional }\end{array}$ & No \\
\hline
\end{tabular}

Fuente: elaboración propia

${ }^{2}$ Se puede consultar la historia fidedigna de la ley en el siguiente link: https://www.bcn.cl/historiadelaley/nc/historia-de-la-ley/5621/ fecha de consulta, 28 de agosto de 2019. ${ }^{3} \mathrm{El} 2007$ con la ley 20.160 se modificó nuevamente el texto del artículo 42, pero referido siempre a las divulgaciones inocuas. 
Como corolario a esta evolución, nos cabe señalar que el Acuerdo ADPIC señala en su art. 33 referido a la "Duración de la protección" que "La protección conferida por una patente no expirará antes de que haya transcurrido un período de 20 años contados desde la fecha de presentación de la solicitud". A dicho respecto, cabe señalar que no se establece con claridad desde que momento se iniciaría la referida protección. Además, dicho artículo contiene una nota signada con el número 8 que dispone: "Queda entendido que los Miembros que no dispongan de un sistema de concesión inicial podrán establecer que la duración de la protección se computará a partir de la fecha de presentación de solicitud ante el sistema que otorgue la concesión inicial". En dicho sentido, es dable interpretar que existe la facultad para incorporar este tipo de protección.

Por otra parte, el art 62, párrafo $2^{\circ}$ establece que "Cuando la adquisición de un derecho de propiedad intelectual esté condicionada al otorgamiento o registro de tal derecho, los Miembros se asegurarán de que los procedimientos correspondientes, siempre que se cumplan las condiciones sustantivas para la adquisición del derecho, permitan su otorgamiento o registro dentro de un período razonable, a fin de evitar que el período de protección se acorte injustificadamente”. El cual también da pie de manera tibia a la posibilidad de protegerlos provisionalmente. En síntesis, el argumento esgrimido para haber eliminado la patente provisional pareciera no ser tal.

\section{Propiedad industrial y patentes}

La propiedad industrial tiene por objeto bienes inmateriales que como señala Gómez Segade (como se citó en Metke, 2001, p. 20) son "creaciones de la mente humana que, mediante los medios adecuados, se hacen perceptibles y utilizables en las relaciones sociales y que por su especial importancia económica son objeto de una tutela jurídica especial." Stiglitz (2008, p. 1703) considera que la propiedad intelectual, a diferencia de la propiedad sobre la tierra, no puede ser definida precisamente según límites geográficos, y que no existen contornos naturales para la propiedad intelectual. Agrega que normalmente los derechos de propiedad se sostienen como un medio para lograr la eficiencia económica; pero que, por el contrario, los derechos de propiedad intelectual resultan en una ineficiencia estática que solo puede justificarse por los incentivos dinámicos. Hay ejemplos que sugieren que las ineficiencias estáticas pueden ser mayores de lo que a menudo se piensa.

Ricardo Sandoval López (2018, p.128) señala que la patente "es un derecho de propiedad industrial, concedido por el Estado a un inventor o a sus herederos, que le permiten hacer uso exclusivo e impedir que otras personas puedan hacer uso o explotar la tecnología o invención patentada." Para Marcelo D 'Amore (2015, p. 108), una patente de invención "es un bien intangible representado por medio de un título de propiedad". Esta lo que otorga es un "derecho negativo", la facultad de "poder excluir a terceros del uso de la invención patentada”. D 'Amore considera a la patente de invención como el más fuerte de los derechos de propiedad intelectual en términos de poder exclusorio, pues da "un monopolio legal respecto de la invención reivindicada", lo que significa que por una parte tiene facultades impeditivas para su uso por terceros, y por otra, imposibilita el reconocimiento legal de la invención por un tercero aun cuando haya podido arribar a la invención protegida por sus propios medios.

En Chile, la patente se define ex lege en el artículo 31 inciso 21 de la ley 19.039 "Se entiende por patente el derecho exclusivo que concede el Estado para la protección de una invención. Los efectos, obligaciones y limitaciones inherentes a la patente están determinados por esta ley." Como condiciones de fondo se distinguen la novedad, el mérito inventivo y la aplicación industrial. Posner y Landes (2004, p. 294 y ss) señalan que "la justificación convencional para otorgar protección legal a las invenciones en cuanto a obras expresivas es la dificultad que un productor puede encontrar al tratar de recuperar sus costos fijos de investigación y desarrollo cuando el producto o proceso que incorpora una nueva invención es fácilmente copiable”. En cuanto a las ventajas, Sandoval (2018, p. 129) menciona el incentivo al creador que otorga el proteger por un plazo de 20 años la invención, unido a que tendrá el monopolio de la explotación directa o licenciamiento; la expectativa de resultar favorecido con las licencias de explotación en caso de tener éxito comercial la patente; evita el plagio; publicidad del invento lo que favorece el progreso tecnológico; fomento del desarrollo de la industria y el comercio así como la transferencia tecnológica. Del mismo modo señala algunas desventajas: Dificulta la libre difusión de las innovaciones; genera obstáculos monopolistas; dificulta el acceso tecnológico a los países menos desarrollados; desincentiva la investigación durante el extenso período de protección y utilización exclusiva sin necesidad de mejorarla.

\section{Patente provisional}

Las patentes otorgan exclusividad desde que son otorgadas, por lo que antes de ello no se contaría con un derecho, sino que con una mera expectativa (Cabanellas, 2004, p. 670). La importancia estriba en una cuestión de timing: los trámites suelen tomar algún tiempo, y el período inmediatamente posterior a la invención suele ser el más importante en términos económicos para su explotación, de tal manera que la falta de protección en este período se contituye en una dificultad a sortear por los "titulares" que efectivamente aun no lo son.

Entre los diferentes tipos de patentes, destaca la patente provisional o precaucional. La finalidad que persigue es la de "proteger los derechos del inventor durante el período que media entre la presentación de la solicitud de una patente y el otorgamiento de esta" como señala Cabanellas (2004, p.669)

Las patentes precaucionales se otorgan con un examen formal o poco más que formal de la solicitud presentada al efecto, dando a su titular derecho a que no se otorguen patentes respecto del objeto de la patente precaucional durante el período determinado sin previa notificación al titular de la patente precaucional, dandole oportunidad de formular oposiciones contra las solicitudes que se presenten sobre el mismo objeto de la patente que detenta.

En Chile, el tratadista Santiago Larraguibel Zavala (1995, p. 216217) plantea que la patente precaucional "se trata de un amparo transitorio cuando un inventor necesita practicar experiencia que lo obliguen a hacer pública su idea, y así evitar la pérdida de la 
novedad..., lo que haría no patentable la invención”. Para GarcíaHuidobro (1992, p. 159) su objetivo no es otro que el de "preservar incólume el derecho del creador más que conjurar el riesgo de una eventual usurpación".
Entre las críticas se expone que su finalidad de protección en el interregno no sería completamente eficiente. Lo anterior, toda vez que perfectamente pueden servir para impedir el aprovechamiento de un tercero que ha tenido acceso ilimitado o ilícito a dicha patente, transformándose precisamente en barreras para la obtención de la patente por el inventor. (Cabanellas, 2004, p. 671)

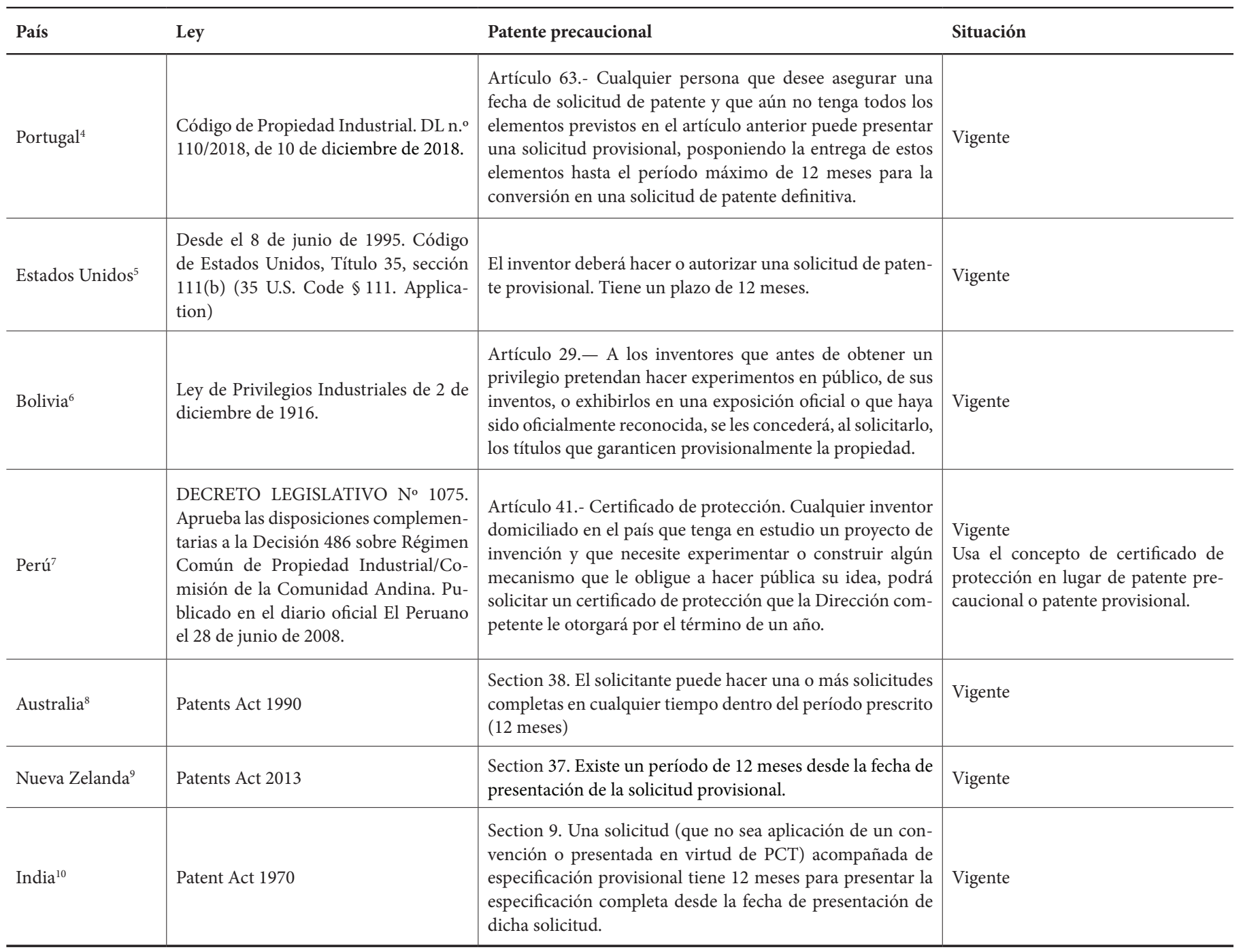

Fuente: elaboración propia

${ }^{4}$ Disponible en: https://wipolex.wipo.int/en/text/508629 fecha de consulta: 25 de agosto de 2019

${ }^{5}$ Disponible en: https://www.law.cornell.edu/uscode/text/35/111 fecha de consulta 23 de agosto de 2019

${ }^{6}$ Disponible en: http://www.silep.gob.bo/silep/masterley/126693 fecha de consulta 23 de agosto de 2019

${ }^{7}$ Disponible en: https://www.indecopi.gob.pe/documents/20791/199826/DecLesg1075/52873b29-90f9-b411-e808-cf2293ff727b fecha de consulta 25 de agosto de 2019

${ }^{8}$ Diponible en: https://www.legislation.gov.au/Details/C2019C00088 fecha de consulta 25 de Agosto 2021

${ }^{9}$ Disponible en: https://www.legislation.govt.nz/act/public/2013/0068/latest/whole.html fecha de consulta 26 de Agosto de 2021

${ }^{10}$ Disponible en: https://ipindia.gov.in/writereaddata/Portal/IPOAct/1_31_1_patent-act-1970-11 march2015.pdf fecha de consulta 26 de agosto de 2021. 
En Argentina y El Salvador existieron patentes precaucionales, pero las leyes que las permitían han sido derogadas, por lo que no tienen aplicación en la actualidad.

\begin{tabular}{l|l|l|l|l|l}
\hline País & Nombre & Artículo & Denominación & Duración y prórroga & Estado actual \\
\hline Argentina $^{11}$ & $\begin{array}{l}\text { Decreto 590/95Ley de pa- } \\
\text { tentes de invención y mo- } \\
\text { delos de utilidad }\end{array}$ & Artículo 80 & Patente precaucional & $\begin{array}{l}1 \text { año prorrogable } \\
\text { cada vez que se venza }\end{array}$ & $\begin{array}{l}\text { Derogado. } \\
\text { Ley No 24.481 de patentes de in- } \\
\text { vencion y modelos de utilidad }\end{array}$ \\
\hline El Salvador & $\begin{array}{l}\text { Ley de patentes de inven- } \\
\text { ción de 19 de mayo de } \\
1913 .\end{array}$ & Artículos 40 a 47 & Patente precaucional & $\begin{array}{l}\text { 1 año. Puede ser re- } \\
\text { novada por una sola } \\
\text { vez. }\end{array}$ & $\begin{array}{l}\text { Derogada por Ley de propiedad } \\
\text { intelectual Decreto 604 de 15 de } \\
\text { julio de 1993 }\end{array}$ \\
\hline
\end{tabular}

Fuente: elaboración propia

\section{El restablecimiento de las patentes precaucionales en la ley chilena.}

El 2 de octubre de 2018 ingresó por Mensaje presidencial a la Cámara de Diputados, el denominado proyecto de "ley corta de INAPI"13, identificado con el boletín 12135-03, hoy ley 21.335, que "Modifica la ley $\mathrm{N}^{\circ} 19.039$, de Propiedad Industrial, la ley $\mathrm{N}^{\circ} 20.254$, que Establece el Instituto Nacional de Propiedad Industrial y el Código Procesal Penal". El concepto de ley corta es una expresión no reconocida en el ordenamiento jurídico de Chile, pero que se ha instalado en el lenguaje del legislador para designar un tipo de proyecto de ley que aborda un tema en aspectos puntuales sobre los que hay menos controversia, y que augura una tramitación más rápida. En materia de modernización del sistema de propiedad industrial la "ley larga" fue propuesta en el año 2013 y está sin avance desde el año 2015. En el caso de la "ley corta de INAPI", como señala el Mensaje presidencial que la propone,$^{14}$ nuestra legislación vigente no contempla la posibilidad de obtener una patente provisional que permita durante un lapso breve hacer reserva del derecho a solicitarla formalmente. Como argumento, el "Informe de Productividad Proyecto de Ley Corta INAPI" elaborado por el Ministerio de Economía Fomento y Turismo fechado en septiembre de 2018 y anexado al Mensaje, da cuenta de que "uno de los mayores problemas es que existen proyectos inmaduros donde los inventores aún no tienen toda la información necesaria para hacer una solicitud formal de patente, pero tienen la necesidad de preservar con urgencia su contenido" (p. 24).
Una de las razones que se aduce para reestablecer las patentes provisorias es el interés que reviste para las Universidades. Al respecto Rojas (2007, p.6) plantea siguiendo a Thiers, que se podría concluir que los objetivos de la Universidad son continuar su labor educativa, y proteger los resultados de I+D que tienen valor comercial. En dicho sentido y atendidas las restricciones presupuestarias que padecen la mayoría de las universidades es que las patentes provisorias cobran sentido estratégico.

Durante la tramitación legislativa, en el trabajo en Comisiones se informa el proyecto para ilustrar la decisión de la Cámara (o Senado) proponiendo el texto definitivo del proyecto de ley a la Sala. Las Comisiones están mandatadas por los artículos 22 de la Ley Organica Constitucional del Congreso, 218 del Reglamento de la Cámara de Diputados y 38 a 39 del Reglamento del Senado, a reunir los antecedentes que estimen necesarios para informar, pudiendo solicitar a las autoridades la comparecencia de funcionarios, asesorías de especialistas, antecedentes, requerir informes, asesoría externa, de la oficina de informaciones y de la Biblioteca del Congreso Nacional, y por supuesto oír a las instituciones y personas que consideren relevante cuyas opiniones aportan al debate (Bronfman et al, 2013, p. 141).

\section{Análisis de la tramitación}

Como primer comentario, cabe señalar que el análisis de la tramitación, no solo reviste interés histórico o científico para los jurista interesados en las materias específicas. También de acuerdo al inciso

\footnotetext{
${ }^{11}$ Disponible en: hhttp://servicios.infoleg.gob.ar/infolegInternet/anexos/35000-39999/35001/texact.htm fecha de consulta 25 de agosto de 2019

${ }^{12}$ Disponible en: http://www.csj.gob.sv/BVirtual.nsf/3db6532d39e032fd06256b3e006d8a73/c7dd02a14343cbf3062577bc005c962b?OpenDocument fecha de consulta: 28 de agosto de 2019 .

${ }^{13}$ Se le llama "ley corta" toda vez que se pretende una ágil tramitación que permita destrabar el Mensaje presidencial identificado con el boletín 8907-03 que "Sustituye las leyes $N^{o} 19.039$, sobre propiedad industrial y $N^{\circ} 20.254$, que crea el Instituto Nacional de Propiedad Industrial", ingresado el 29 de abril de 2013, sin movimiento desde el mes de febrero de 2015. Aún se encuentra en el primer trámite constitucional del Senado. Dicha iniciativa no se refiere a las patentes provisionales o precaucionales. No obstante, el Mensaje sí señala el fundamento tenido en consideración para su eliminación: “Como consecuencia de los acuerdos internacionales adoptados por Chile, la ley N 19.039 ha sido objeto de importantes modificaciones. En el año 2005, la ley No 19.996 introduce enmiendas con el objeto de adecuar la legislación nacional al Acuerdo de los Aspectos de la Propiedad Intelectual relativos al Comercio (ADPIC), cuyas reformas más relevantes fueron la derogación de las patentes precaucionales y de reválida”. Disponible en: https://www. camara.cl/pley/pley_detalle.aspx?prmID=9311\&prmBoletin=8907-03 Fecha de consulta: 28 de agosto de 2019.

${ }^{14}$ Disponible en: https://www.camara.cl/pley/pley_detalle.aspx?prmID=12653\&prmBoletin=12135-03 fecha de consulta: 25 de agosto de 2019.
} 
segundo del artículo 19 del Código Civil, constituye un elemento para "interpretar una expresión obscura de la ley" al recurrir "a su intención o espíritu claramente manifestada en ella misma (la ley) o en la historia fidedigna de su establecimiento".

Tanto en el Mensaje, como en el Primer informe de la Comisión de Economía, Fomento, Micro, Pequeña y Mediana Empresa, Protección de los consumidores y Turismo, fechado 11 de enero de 2019, se planteó que la patente provisional iría en beneficio de las universidades y pequeños emprendedores. En dicho informe, la Directora Nacional (s) de INAPI, Carolina Belmar señaló que las patentes provisionales son figuras "muy usadas en derecho comparado y que aparece de gran utilidad en Chile". Ello pues permite al inventor presentar la solicitud sin cumplir con la totalidad de las formalidades para poder gozar de la fecha original de presentación teniendo un plazo de un año para ingresar formalmente el formulario completo (pp. 20-21 y 36-37).

El invitado Juan Pablo Egaña asesor en materias de Propiedad Intelectual de la Sociedad de Fomento Fabril. (SOFOFA) ${ }^{15}$ manifestó que los inventores tendrían la posibilidad "de entrar con una solicitud básica, sin intervención de estudios jurídicos, para fijar una fecha prioritaria y poder juntar recursos que un año después permitan presentar una solicitud regular" (pp 24-25). El invitado Carlos Harms, abogado encargado de Estudios de Asociación de Emprendedores de Chile (ASE$\mathrm{CH})$ comentó el caso de unas personas "que desarrollaron una invención, pero los costos del trámite en Inapi eran altísimos. Concurren a Corfo que les exige el producto mínimo viable y cuando estuvieron en condiciones de inscribirlo en Inapi, les señalaron que ese invento ya se estaba desarrollando y comercializando, no es innovador y no tienen prioridad". (p. 33)

En la Cámara de diputados, fueron presentadas tres indicaciones al proyecto, dos que finalmente fueron retiradas, y otra aprobada por unanimidad para eliminar la frase "de tal manera que la invención pueda llevarse a cabo por una persona experta en la técnica” (p. 67). Una vez aprobado en dicha Cámara, el proyecto pasó al Senado, en cuya Comisión de Economía en sesión de 10 de julio de 2019, la Directora del Instituto Nacional de Propiedad Industrial, señora Loreto Bresky presentó el Proyecto. La Directora señaló que se reestablece la patente provisional fijando una invención "con un puntito rojo mientras en paralelo se ve la factibilidad, ensayos, mercados, preparación de documentación y así, la persona no se embarca en un proceso de solicitud de patente si no tiene esa claridad”. Además señaló que esta medida apunta principalmente a las Universidades (p. 14). En sesión de 24 de julio de 2019 expusieron los Directores de la Asociación Chilena de la Propiedad Industrial (ACHIPI) Marcelo Correa y Eduardo Molina quienes al referirse a la materia en comento señalaron que "este articulo tal como se encuentra redactado dará pie a confusiones y exigencias incumplibles, dado que, por un lado, su espíritu es permitir la presentación de invenciones que aún no se encuentran totalmente desarrolladas, pero, por otro lado, exige que el documento describa la invención de manera suficientemente clara y completa, lo que no es posible por su naturaleza de provisional." Por ello proponen que el proyecto tenga una redacción más precisa en este punto (p. 18-19). Los directores de ACHIPI proponen modificaciones en los incisos $4^{\circ}$ agregando la frase "atendido el propósito de la misma" que contextualiza la naturaleza de la patente provisional; y $5^{\circ}$ donde agregan la frase " $y$ continuará su tramitación en el mismo expediente administrativo de la solicitud provisional" que parece ser correcta en cuanto a la economía procesal. Durante la tramitación en el Senado, en la sesión $\mathrm{N}^{\circ} 48$, de fecha 11 de septiembre de 2019, el senador Rodrigo Galilea se refirió a este tipo de patente, considerando que fortalece las posibilidades de financiamiento a los inventores nacionales y que contribuyen "al objeto de que un inventor tenga la posibilidad de patentar y disponer de tiempo para terminar después su investigación." (pps. 52 y 53). Este proyecto de ley ha concluido su tramitación en el Congreso y con fecha 5 de julio de 2021 fue publicado en el Diario Oficial, contemplando un nuevo artículo $40^{16}$ que reestablece la patente provisional en nuestro sistema.

Esta ley tiene una entrada en vigencia diferida, que depende de la publicación del reglamento de esta ley en el Diario Oficial. Ese reglamento lo debe dictar el Presidente de la República en un plazo de seis meses contado desde 5 de julio de 2021, mediante decreto supremo expedido por el Ministerio de Economía, Fomento y Turismo. Una vez que ese reglamento haya sido publicado los investigadores podrán acogerse a la protección y beneficios que otorga la patente provisional.

${ }^{15}$ Principal entidad del gremio empresarial de Chile que de acuerdo a su web, agrupa el 100\% de la actividad industrial en Chile. Disponible en: https://web.sofofa.cl/nosotros/ que-es-sofofa/ fecha de consulta 25 de agosto de 2019.

16“Artículo 40.- Sin perjuicio de lo establecido en el artículo 42, cualquier persona que tenga una invención, pero que aún no pueda cumplir con todos los elementos de una solicitud de patente para su presentación, de acuerdo a lo establecido en el artículo 43, podrá presentar una solicitud de patente provisional, que el Instituto reconocerá por el término de doce meses, previo pago de la tasa correspondiente.

La solicitud de patente provisional confiere a su titular un derecho de prioridad por el plazo señalado en el inciso anterior, contado desde su presentación. La solicitud provisional no podrá reivindicar la prioridad de una solicitud anterior.

La solicitud de patente provisional no requiere de la presentación de reivindicaciones ni de las declaraciones a que se refiere el artículo 44, sin perjuicio de lo que establezca el reglamento.

Además, la solicitud de patente provisional deberá venir acompañada de un documento en español o inglés que describa la invención de manera suficientemente clara y completa. En caso de ser necesario deberá acompañarse también, al menos, un dibujo

Antes de la expiración del plazo de doce meses contado desde la fecha de presentación de la solicitud provisional, el titular deberá solicitar la patente definitiva, acompañando todos los documentos mencionados en los artículos 43, 43 bis y 44, debidamente redactados en español.

Si transcurrido el plazo señalado en el inciso anterior, el titular de una patente provisional no hubiese solicitado la patente definitiva, se tendrá por no presentada.

La solicitud definitiva conservará la prioridad de la solicitud provisional, siempre que su contenido no implique una ampliación del campo de la invención de esta última o de la divulgación contenida en la solicitud provisional. Si la solicitud definitiva ampliare dicho campo, los contenidos modificados tendrán para todos los efectos jurídicos la fecha de presentación de la solicitud definitiva.

El plazo de vigencia de la solicitud de patente definitiva presentada de acuerdo a los artículos precedentes se contará desde la fecha de presentación de la solicitud provisional de patente." 


\section{Conclusiones}

1.- Del análisis de la historia de las leyes, se concluye que los argumentos para eliminar dicha institución se refirieron a compromisos supuestamente adquiridos con la comunidad internacional, como se señaló en mensaje presidencial que la derogó. Durante la misma tramitación de la ley que la eliminó no hubo debate sobre este tipo de patente. Fue eliminada sin mediar argumentos a favor ni en contra.

2.- Las leyes de propiedad industrial de diferentes países de América Latina incorporaron este tipo de patente desde finales del siglo XIX y comienzos del siglo XX. Sin embargo, hacia finales del siglo pasado hubo una tendencia para eliminarla. Chile fue parte de ella, en un proyecto de ley iniciado en 1999 y finalizado en 2005. Trece años después, se ha intentado restablecerla. Lo llamativo del caso es que las leyes de propiedad industrial de Estados Unidos de América, que nunca la contemplaron, sí lo hicieron a contar de 1995, cuando se incorporó a su legislación federal la posibilidad de solicitar provisionalmente una patente.

3.- El artículo 42 de la ley chilena de Propiedad Industrial, en su redacción actual, contempla la divulgación inocua. Este concepto constituye una excepción al requisito de novedad que debe cumplir toda patente de invención, ya que permite que se pueda dar a conocer una invención antes de la solicitud de la patente. La protección es pasiva porque se limita a no perjudicar el carácter de novedoso. La diferencia con la patente precaucional radica en que esta última tiene un carácter activo, ya que debe proteger al inventor que la ha obtenido en contra de otro que pretende patentar una invención idéntica a la protegida. Por ello, la patente provisional funciona y se entiende en relación con el derecho de prioridad que otorga.

4.- En la tramitación de la ley 21.355 no se esgrimieron razones de peso para reintegrar esta figura. A este último respecto, consideramos que la patente provisional o precaucional constituye un incentivo a los inventores en general, pero particularmente a las instituciones de educación superior, como universidades y centros de investigación, para poder proteger de manera transitoria aquellas innovaciones que requieren el cumplimiento de una serie de requisitos para obtenerse, en definitiva.

5.- La ley 21.335 restablece la patente precaucional, llamándola patente provisional. Lo importante es que confiere a su titular un derecho de prioridad, que dura un año. Esto es una manifestación del principio "Prior in tempore, potior in iure", o el anglosajón "First in time, first in right" primero en el tiempo, mejor en el derecho. Debemos entender que esta prioridad se ejercerá sobre cualquier otro que pretenda patentar una invención idéntica. El procedimiento para que el inventor que ha obtenido esta patente provisional pueda actuar en contra de quien pretenda registrar una idéntica, debe ser el mismo que se aplica para la protección de las patentes definitivas.

6.- Al restablecer de la patente provisional cualquier persona que tenga una invención susceptible de ser patentada deberá estar atenta a solicitar este tipo de patente, teniendo presente que, una vez realizada esa gestión, dispondrá de doce meses para solicitar la patente definitiva. En caso de no obtener la solicitud definitiva dentro de ese plazo, que es improrrogable, caducará su patente provisional y su solicitud se tendrá por no presentada. Este proyecto, a diferencia de las normativas anteriores, no establece una norma expresa que disponga que la invención cuyo patentamiento ha fallado pasa a ser de uso común, o de dominio público. Esa falta de claridad puede provocar que, si un inventor obtiene una patente provisional y luego no puede obtener la patente definitiva, quedaría expuesto a que se considere que su invento no cumple con el requisito de novedad. Este eventual problema podría subsanarse con una interpretación extensiva de las normas sobre divulgación inocua, pero el proyecto no se refiere a ello.

7.- Del análisis tanto de la regulación previa, de la discusión legislativa, como de las normas comparadas y el procedimiento, nos es dable destacar las siguientes ventajas: i) el que puede gozarse tempranamente de la protección de la innovación con ii) requisitos más sencillos que una patente definitiva, puesto que iii) no es necesario cumplir con todos los requisitos formales de una solicitud de patente, como las reivindicaciones ni las declaraciones del artículo 44 (novedad, propiedad y utilidad de la invención), bastando solo describir de manera suficientemente clara y completa la invención, lo que implica iv) un coste económico menor. La patente provisional otorga fecha de presentación y derecho de prioridad a la invención, v) resguardándole durante el término de doce meses que señala el nuevo artículo 40 para que vi) pueda, vincularse con empresas u otros socios, vii) apalancar fondos, o viii) hacer prospección comercial. También es útil toda vez que ix) permite la realización de experimentos que permitan dilucidar la efectividad científico-técnica. Permite x) estudiar la viabilidad comercial, además del xi) posicionamiento temprano mediante el marketing. En definitiva le da la opción al inventor para que pueda evaluar el persistir en la tramitación de la patente definitiva.

A la fecha de publicación de esta contribución académica, no se ha publicado el Reglamento, mismo que conforme al artículo $8^{\circ}$ transitorio de la ley 21.335 le otorgará vigencia a la ley, y para lo cual existe un plazo de seis meses desde su publicación que fue el 05 de julio de 2021, de acuerdo al artículo $7^{\circ}$ transitorio. Es de esperar que dicho Reglamento complemente adecuadamente la ley clarificando adecuadamente los puntos aquí señalados.

\section{Referencias}

Botana Agra, M. (2017) Invención y patente. En Fernández-Nóvoa, C., Otero Lastres, J., Botana Agra, M., Manual de Propiedad Industrial. Tercera Edición. (pp. 99-112). Madrid: Marcial Pons.

Burgos, F. (2014) Innovación. Su promoción a través de la Propiedad Intelectual, subsidios e incentivos Tributarios. Santiago: Legal Publishing.

Bercovitz, A. (2017) Apuntes de Derecho Mercantil. Derecho mercantil, Derecho de la competencia y propiedad industrial. $18^{a}$ edición. Cizur Menor: Thomson Aranzadi.

Bronfman, A., Cordero, E., Aldunate, E. (2013). Derecho Parlamentario chileno. Funciones y atribuciones de Congreso Nacional. Santiago: Legal Publishing. 
Cabanellas, G. (2004). Derecho de las patentes de invención. Tomo 1. Buenos Aires: Heliasta.

Chang, H. (2014, p. 196) Economics. The User's Guide. New York: Bloomsbury.

Claro Solar, L (1992) Explicaciones de Derecho Civil chileno y comparado. Tomo sexto. De los Bienes. Santiago: Editorial Jurídica de Chile.

Clarke, R. (1993). Economía Industrial. Madrid: Colegio de Economístas de Madrid.

Coloma, G. (2006). Análisis Económico de los Derechos de propiedad. En Kluger, V. Análisis Económico del Derecho. (pp. 175-198). Buenos Aires: Heliasta.

Cooter, R. \& Ulen, T. (2016) Derecho y Economía. Ciudad de México: Fondo de Cultura Económica.

Cortes, M. (2012). Patentes de invención. Aspectos jurídicos. Santiago: Thomson Reuters.

D 'Amore, M. (2015). Defensa de la competencia y propiedad intelectual. Buenos Aires: BdeF.

De Souza Querido et al. (2011). What is the Destiny of Patents of Brazilian Universities? Journal of Technology Management \& Innovation, 6(1), 46-57. http://dx.doi.org/10.4067/S0718-27242011000100005

García-Huidobro, V (1992) Legislación sobre propiedad industrial. Análisis, Jurisprudencia y guía práctica. Santiago: Editorial Jurídica de Chile.

Landes, W.M, \& Posner, R.A. (2003). The Economic Structure of Intellectual Property Law. Cambridge, MA: The Belknap Press of Harvard University Press.
Larraguibel, S. (1979) Derecho de autor y propiedad industrial. Nuevas disposiciones constitucionales. Santiago: Editorial Jurídica de Chile.

Larraguibel, S. (1995). Tratado sobre la propiedad industrial. Santiago: Editorial Jurídica Conosur.

Metke, R. (2001) Lecciones de propiedad industrial. Medellín: Diké. Rojas, J. (2007). Capturando Valor en las Universidades y Centros Tecnológicos. Hacia la definición de estrategias, políticas y procedimientos de apropiabilidad, protección legal, explotación y transferencia tecnológica de resultados de proyectos de I+D+I en Chile. Journal of Technology Management \& Innovation, 2(1), 4-10

Ruiz-Tagle, P. (2004). Propiedad intelectual y contratos. Santiago: Editorial Jurídica de Chile.

Samuelson, p \& Nordhaus, W. (2010). Economía con aplicaciones a Latinoamérica- Decimonovena edición. México: McGraw-Hill.

Sandoval, R. (2018) Derecho Comercial Tomo III. Propiedad Industrial, marcas, nombres de dominio, patentes, arbitraje comercial internacional. Santiago: Editorial Jurídica de Chile.

Stiglitz. J. (2008). Economic Foundations of Intellectual Property Rights. Duke Law Journal, 57(6),1693-1724. https://doi.org/10.7916/ D8474M9P

Tirole, J. (2017). La Economía del bien común. Barcelona: Taurus.

Vodanovic. A. (1993) Repertorio de Legislación y Jurisprudencia chilenas. Propiedad Industrial. Prenda Industrial. Prenda agraria. Compraventa de cosas muebles a plazo. Santiago: Editorial Jurídica de Chile 
